\title{
Administration of antenatal corticosteroids for singleton preterm birth in China, 2017 to 2018
}

\section{Qing Wang}

Tongji University School of Medicine

\section{Siyuan Jiang}

Children's Hospital of Fudan University

\section{Xuefeng Hu}

Tongji University School of Medicine

\section{Chao Chen}

Children's Hospital of Fudan University

\section{Yun Cao}

Children's Hospital of Fudan University

\section{Shoo Kim Lee}

University of Toronto

Jiang-Qin Liu ( $\square$ jiangqinliu@163.com )

Tongji University School of Medicine

\section{Research Article}

Keywords: antenatal corticosteroids, preterm, perinatal factors, neonatal mortality, bronchopulmonary dysplasia

Posted Date: September 30th, 2021

DOI: https://doi.org/10.21203/rs.3.rs-941860/v1

License: (c) (i) This work is licensed under a Creative Commons Attribution 4.0 International License. Read Full License 


\section{Abstract \\ Background}

The administration of antenatal corticosteroids (ACS) to women who are at risk of preterm birth has been proven to reduce not only the mortality, but also the major morbidities of the preterm infants. The rate of ACS and the risk factors associated with ACS use in Chinese population is unclear. This study aimed to investigate the rate of ACS use and the associated perinatal factors in the tertiary maternal centers of China.

\section{Methods}

Data for this retrospective observational study came from a clinical database of preterm infants established by REIN-EPIQ trial. All infants born at $<34$ weeks of gestation and admitted to 18 tertiary maternal centers in China from 2017 to 2018 were enrolled. Any dose of dexamethasone was given prior to preterm delivery was recorded and the associated perinatal factors were analyzed.

\section{Results}

The rate of ACS exposure in this population was $71.2 \%$ (range $20.2 \%-92 \%$ ) and the ACS use in these 18 maternal centers varied from $20.2-92.0 \%$ in this period. ACS exposure was higher among women with preeclampsia, caesarean section delivery, antibiotic treatment and who delivered infants with lower gestational age and small for gestational age. ACS use was highest in the 28-31 weeks gestational age group, and lowest in the under 26 weeks of gestational age group $\left(x^{2}=65.478, P<0.001\right)$. ACS exposure was associated with lower odds of bronchopulmonary dysplasia or death (OR, $0.778 ; 95 \% \mathrm{Cl} 0.661$ to $0.916)$ and invasive respiration requirement $(\mathrm{OR}, 0.668 ; 95 \% \mathrm{Cl} 0.585$ to 0.762$)$ in this population.

\section{Conclusion}

The ACS exposure was variable among maternity hospitals and quality improvement of ACS administration is warranted.

\section{Backgrounds}

Preterm birth has been increasing in China in recent decades, and accounted for $6.9 \%$ of live birth or 1.1 million preterm infants annually in $2019^{1}$. Preterm birth is a leading cause of neonatal mortality in China, second only to perinatal asphyxia ${ }^{2}$. Consequently, management of preterm birth and improvement of preterm birth outcomes is a priority for China. 
Administration of antenatal corticosteroids (ACS) to women who are at risk of preterm birth has been proven to decrease the mortality of preterm infants and reduce not only major morbidities like neonatal respiratory distress syndrome (NRDS), necrotizing enterocolitis (NEC) and intraventricular hemorrhage (IVH), but also improve long term developmental outcomes ${ }^{3}$. ACS has been widely accepted as standard of care for anticipated preterm deliveries between 24 to 34 weeks of gestational age $\mathrm{e}^{4,5}$. The best timing of the ACS is within 7 days of and prior to premature delivery ${ }^{6}$. One repeat course is recommended for pregnant women below 34 weeks of gestational age who have received one prior course of ACS for risk of preterm delivery and more than 2 repeated courses of ACS are not recommended ${ }^{7}$. ACS is safe for pregnant women ${ }^{8}$. Nevertheless, the prevalence of ACS administration varies in different gestational ages and different maternal centers and is reported to be between $70-90 \%$ among pregnant women less than 34 weeks of gestational age in high income countries ${ }^{9,10}$ and $50-53 \%$ in China ${ }^{11,12}$. This gap merits investigation and needs to be reduced to improve the care of preterm infants. In this study, we aim to analyze the use of ACS among tertiary level maternity and infant health centers in China, to gain insights that may facilitate development of a strategy of quality improvement to increase ACS use.

\section{Methods}

\section{Overview}

Data for this retrospective observational study came from a clinical database of preterm infants established by REIN-EPIQ (REduction of Infection in Neonatal intensive care units using the Evidencebased Practice for Improving Quality) trial (REIN-EPIQ study, clinicaltrials.gov \#NCT02600195) ${ }^{13}$. The study was reviewed by the ethics committee of Children's Hospital of Fudan University. The consent from the parents was waivered by the ethical committee regarding to the retrospective data abstraction from each hospital. REIN-EPIQ collected standardized maternal and infant data from 25 tertiary level neonatal intensive care units (NICU), including 18 maternity hospitals and 7 children's hospitals from May 2015 to April 2018 for the purpose of quality improvement for managing infection and antibiotic use in level III NICUs in China.

\section{Population}

The subjects were preterm infants whose gestational age was less than 34 weeks. The inclusion criteria for the study were: (1) gestational age < 34 weeks; (2) birth weight < 1500g; (3) admission to the NICU of member hospitals of REIN-EPIQ within 7 days of birth; (4) discharge time from May 1, 2015 to April 30, 2018. Exclusion criteria were: children with congenital malformations, including severe organ structural malformations and chromosomal abnormalities.

A total of 27,534 children were included in the REIN-EPIQ database during this period. Only inborn preterm infants were included in this study because there was a high incidence of missing perinatal data among outborn infants. Consequently all 7 children's hospitals were excluded. We excluded data prior to 2017 because twins were not identified prior to that time. 
During 2017 and 2018, there were 10,598 singleton preterm infants below 34 weeks of GA admitted into the 18 participating maternity hospital NICUs. We excluded 1529 out-born infants as well as 180 in-born infants with missing information on ACS use. The remaining 8,889 infants were included in the analysis. Of these, 636 infants were discharged against medical advice. (Figure 1).

\section{Data collection and data quality control}

A standardized database was used for data collection, including maternal and infant baseline data, and information on clinical care and outcomes of infants. A trained and dedicated data abstractor collected data at each site using standardized data definitions established prior to study commencement. Data were uploaded monthly to the research center of Children's Hospital of Fudan University, and data integrity and quality were checked by the research center.

\section{Measures and definitions:}

ACS administration was defined as any dose of dexamethasone administration prior to preterm delivery. Whether the ACS course was complete or incomplete was not identified in this database. Hypertensive disorders of pregnancy (HDP) was defined as either chronic hypertension (persistent elevation of blood pressure before 20 weeks of gestation or prior to pregnancy) or pregnancy-induced hypertension if blood pressure $>145 / 95$ was first recorded after 20 weeks of gestation. Prolonged premature rupture of membrane (PROM) was defined as membrane rupture more than 6 hours before the onset of regular spontaneous uterine contractions. Prenatal antibiotics was defined as administration of antibiotics during second and third trimester of pregnancy. The Transport Risk Index of Physiologic Stability (TRIPS) score was used as an illness severity score on NICU admission ${ }^{14}$. Bronchopulmonary dysplasia (BPD) was defined as mechanical ventilation or oxygen dependency at 36 weeks of postmenstrual age or discharge ${ }^{15}$. NEC was defined as $\geq$ stage 2 according to Bell criteria ${ }^{16}$. IVH was defined as $\geq$ grade 3 according to Papile criteria. Periventricular leukomalacia (PVL) was defined as the presence of periventricular cysts on cranial ultrasound or cranial magnetic resonance imaging scans ${ }^{17}$. Early-onset sepsis (EOS) was defined as the presence of clinical symptoms and a positive culture from blood or cerebrospinal fluid samples drawn within 72 hours after birth ${ }^{18}$. Retinopathy of prematurity (ROP) was defined as $\geq$ stage 3 according to the International Classification of ROP ${ }^{19}$.

\section{Statistical analysis}

Stata / SE 15.0 software was used for statistical analysis. For normally distributed data, Mean \pm SD, and t-test were used for comparison between groups; for non-normally distributed data, Median (Q1, Q3), and rank sum test were used instead. Count data were expressed as frequency and rate, and the $\chi 2$ test or Fisher exact probability method were used for comparison between groups. Logistic multiple regression analysis was used to analyze for risk factors associated with ACS. The $\mathrm{P}<0.05$ level of significance was used. 


\section{Results}

The prevalence of ACS use (at least one dose) was 71.2\% (6325/8889). On univariate analysis, women who received ACS prior to delivery were more likely to have regular antenatal care, HDP, PROM, prenatal antibiotics, and delivery by cesarean section (CS) compared to those with no ACS exposure (Table 1). Infants exposed to ACS during pregnancy had smaller birth weight and gestational age, and were more likely to be SGA and have Apgar score $<4$ at 1 and 5 minutes of life.

Table 1: Univariate analysis of perinatal factors

\begin{tabular}{|llll|}
\hline Variables & ACS n(\%) & No ACS n(\%) & P value \\
\hline $\mathrm{n}$ & 6325 & 2564 & \\
\hline Gestational age (week) & $31.2 \pm 1.9$ & $31.4 \pm 2.1$ & $<0.001$ \\
\hline Birth weight (gram) & $1608 \pm 404$ & $1686 \pm 443$ & $<0.001$ \\
\hline Male & $3562 / 6325(56.3)$ & $1472 / 2564(57.4)$ & 0.346 \\
\hline SGA & $895 / 6325(14.2)$ & $297 / 2564(11.6)$ & 0.001 \\
\hline Primigravida & $1794 / 6321(28.4)$ & $744 / 2564(29.0)$ & 0.548 \\
\hline Regular prenatal care & $6262 / 6317(99.1)$ & $2486 / 2546(97.6)$ & $<0.001$ \\
\hline Preclampsia & $1344 / 6306(21.3)$ & $379 / 2542(14.9)$ & $<0.001$ \\
\hline GDM & $898 / 6306(14.2)$ & $354 / 2544(13.4)$ & 0.691 \\
\hline Cesarean section & $3402 / 5943(57.2)$ & $1201 / 2308(52.0)$ & $<0.001$ \\
\hline PROM>18 hours & $2138 / 6238(34.3)$ & $459 / 2514(18.3)$ & $<0.001$ \\
\hline Maternal Antibiotics & $2421 / 5775(41.9)$ & $469 / 2374(19.8)$ & $<0.001$ \\
\hline 1' Apgar $\leq 3$ & $244 / 6316(3.86)$ & $164 / 2553(6.42)$ & $<0.001$ \\
\hline 5' Apgar $\leq 3$ & $54 / 6209(0.87)$ & $33 / 2451(1.35)$ & 0.045 \\
\hline TRIPS (6300/2558) & $12.5 \pm 10.0$ & $13.2 \pm 11.1$ & 0.006 \\
\hline Death or BPD & $1427 / 12184(11.7)$ & $588 / 4861(12.1)$ & 0.483 \\
\hline Death & $492 / 12184(4.0)$ & $263 / 4861(5.4)$ & $<0.001$ \\
\hline BPD & $1053 / 11058(9.5)$ & $396 / 4442(8.9)$ & 0.240 \\
\hline Invasive Ventilation & $2709 / 12184(22.2)$ & $1231 / 4861(25.3)$ & $<0.001$ \\
\hline
\end{tabular}

ACS: antenatal corticosteroids; SGA: small for gestational age; GDM: gestational diabetes mellitus; PROM: premature rupture of membranes; TRIPS: transport risk index of physiologic stability; BPD: bronchopulmonary dysplasia. 
Logistic regression analysis of perinatal factors showed that factors independently associated with ACS exposure were small for gestational age (SGA), HDP, CS, PROM and prenatal antibiotics. There was no correlation between the use of ACS and infant gender, GDM and primipara (table 2). Multivariate logistic regression also showed that infants exposed to ACS during pregnancy had smaller birth weight and gestational age, and were more likely to be SGA and have Apgar score $<4$ at 1 and 5 minutes of life. ACS exposure was associated with lower odds of BPD or death (OR, 0.778; 95\% $\mathrm{Cl} 0.661$ to 0.916 ), death (OR, $0.608 ; 95 \% \mathrm{Cl} 0.478$ to 0.774$)$, BPD (OR, $0.806 ; 95 \% \mathrm{Cl} 0.679$ to 0.955$)$ and invasive respiration requirement (OR, $0.668 ; 95 \% \mathrm{Cl} 0.585$ to 0.762$)$.

Table 2: Logistic regression of perinatal factors associated with antenatal corticosteroids administration

\begin{tabular}{|lllll|}
\hline Variables & OR & $95 \% \mathrm{Cl}$ & & P value \\
\hline Gestational age & 0.911 & 0.890 & 0.933 & $<0.001$ \\
\hline small for gestational age & 1.241 & 1.083 & 1.423 & 0.002 \\
\hline Male & 0.936 & 0.858 & 1.021 & 0.138 \\
\hline Preeclampsia & 1.628 & 1.432 & 1.851 & $<0.001$ \\
\hline gestational diabetes mellitus & 1.069 & 0.945 & 1.209 & 0.292 \\
\hline Primigravida & 0.978 & 0.894 & 1.071 & 0.633 \\
\hline Cesarean section & 1.347 & 1.224 & 1.482 & $<0.001$ \\
\hline premature rupture of membranes & 1.414 & 1.243 & 1.609 & $<0.001$ \\
\hline Antibiotics & 2.604 & 2.309 & 2.936 & $<0.001$ \\
\hline
\end{tabular}

When stratified by gestational age, the proportion of exposed ACS in infants less than 26 weeks, 26-27 weeks, 28-31 weeks and 32-33 weeks gestational age were $54.1 \%, 65.6 \%, 74.8 \%$ and $68.2 \%$ respectively. ACS use was highest in the 28-31 weeks gestational age group, and lowest in the under 26 weeks gestational age group $\left(x^{2}=65.478, P<0.001\right)$. The incidence of ACS exposure was $70.8 \%$ among infants with BW less than $1000 \mathrm{~g}, 75.2 \%$ among infants with BW between $1000 \mathrm{~g}$ and $1499 \mathrm{~g}, 71.5 \%$ among infants with BW between $1500 \mathrm{~g}$ and $1999 \mathrm{~g}$, and $63.7 \%$ among infants with BW greater than or equal to $2000 \mathrm{~g}$. ACS exposure was highest among infants with BW 1000-1499g, and lowest among infants with BW less than $1000 \mathrm{~g}\left(\mathrm{x}^{2}=71.196, \mathrm{P}<0.001\right)$.

The proportion of ACS use varied from $20.2 \%$ to $92.0 \%$ in these 18 maternal centers. There was significant inter-institutional variation in ACS use for different gestational age groups (Figure 2). The 
proportion of ACS use was positively correlated with the number of the infants (Pearson coefficient 0.487, $p=0.04$ ), and particularly so among very low birth weight infants (Pearson's coefficient $0.524, p=0.03$ ).

\section{Discussion}

Antenatal corticosteroids administration has become an important obstetrical practice for improving the outcomes of preterm infants less than 34 weeks of gestational age since $1972^{8}$. It reduces the risk of neonatal mortality and morbidities including IVH, NEC, and ROP in every gestational age group ${ }^{10}$. ACS use in North America and Europe were reported to be between $70-91.4 \%{ }^{20,21}$, which is significantly higher than the $50-56 \%$ reported previously in China $^{13}$. Although the $71.2 \%$ incidence reported in our study is a significant improvement over previous reports, there is still room for improvement in China.

Administration of ACS to pregnant women at risk of preterm delivery is standard of care for obstetricians in China. Usually a course of intra-muscular dexamethasone (6mg at 12 hours interval for two days) is used in China instead of the betamethasone (12 $\mathrm{mg}$ at 24 hours of interval for two days) used in North America and Europe ${ }^{22}$. Brownfoot et al reported that dexamethasone may be associated with lower incidence of IVH and shorter duration of hospitalization but the data is inconclusive ${ }^{22}$. A more recent study reported no significant difference in outcomes at 2 years of age ${ }^{23}$. Dexamethasone and betamethasone are both safe for pregnant women ${ }^{23}$. Although infants previously exposed to these ACS have an increased risk of long-term adverse neurodevelopmental and neurosensory outcomes when delivered at term ${ }^{24}$, there was no evidence that a single course of ACS increased the risk of metabolic disease long term ${ }^{25}$. However, there may be risks in repeated courses of $\operatorname{ACS}^{26,27}$. It is very challenging for obstetricians to accurately predict whether preterm delivery will occur within one week and when ACS should be optimally administered ${ }^{28}$. Existing tests for predicting preterm birth are inaccurate and can result in missed opportunities for using $\mathrm{ACS}^{29,30}$. In a Japanese report, there was a high chance of missing the ACS for pregnant women who received tocolysis due to the risk of preterm delivery while only $23 \%$ were given $\mathrm{ACS}^{31}$. In our study, the women who had more preterm related complications and who were 28-31 weeks of GA at delivery were more likely to receive ACS. It is possible that increasing the awareness of ACS and developing a standardized protocol may improve the rate of ACS administration to the women at risk of preterm delivery.

\section{Variation of ACS among maternity hospitals in China}

Understanding the reasons for missing ACS in pregnant women less than 34 weeks GA is very important for quality improvement ${ }^{32}$. Regional variations in incidence of ACS administration present an opportunity for improvement. For example, inter-institutional ACS use varied from $23 \%$ to $76 \%$ with an average of $58 \%$ in Canada in 1996-199733. Following a national quality improvement effort, this improved to $91.4 \%$ and inter-institutional variation was significantly reduced ${ }^{34}$. Outcomes of these infants were also significantly improved ${ }^{21}$. Many perinatal collaboratives have worked on quality improvement of ACS administration by focusing on reducing missed opportunities and optimizing the appropriate time of use ${ }^{35}$. By establishing 
a reliable practice culture, Kaplan et al reported that ACS use increased from $76 \%$ at baseline to $86 \% 36$. Similarly, in a report from California from 2005 to 2011 , ACS use was increased from $82 \%$ to $87.9 \%$ with a quality improvement strategy. They also found that a lower level of care was associated with lower incidence of ACS use ${ }^{37}$. Of significance, the ACS use is lower in low and middle income countries, where the majority of preterm death occur ${ }^{38}$. According to the Every Newborn Action Plan report, the use of ACS varies from $4 \%$ to $74 \%$ among low and middle income countries ${ }^{39}$. Therefore, reducing regional differences is a viable strategy for improving ACS use and outcomes of preterm infants.

\section{Limitations}

This is a retrospective study and only singleton births were included. Information on complete versus incomplete course, or multiple courses of ACS was not available. The knowledge level of obstetricians about ACS was not investigated and may present an opportunity for improvement.

\section{In Conclusion}

The overall incidence of ACS use among Chinese level III maternal hospitals in our cohort was $71.2 \%$. The incidence of prenatal ACS use was highest among preterm infants who were 28-31 weeks GA and in pregnancies with medical complications. There are opportunities for improving ACS use in Chinese hospitals.

\section{Abbreviations}

ACS: antenatal corticosteroids;

NRDS: neonatal respiratory distress syndrome;

NEC: necrotizing enterocolitis;

IVH: intraventricular hemorrhage;

REIN-EPIQ: REduction of Infection in Neonatal intensive care units using the Evidence-based Practice for Improving Quality;

NICU: neonatal intensive care unit;

HDP: hypertensive disorders of pregnancy;

PROM: prolonged premature rupture of membrane;

TRIPS: transport risk index of physiologic stability;

BPD: bronchopulmonary dysplasia; 
PVL: periventricular leukomalacia;

EOS: early-onset sepsis;

ROP: retinopathy of prematurity;

CS: cesarean section;

SGA: small for gestational age;

\section{Declarations}

\section{Authors' Contribution:}

QW and JQL drafted the manuscript. SJ statistically analyzed and interpreted the data. XH acquisited and analyzed the data. SKL substantively revised the manuscript. JQL and CC conceived the work; JQL and $Y C$ designed the study.

\section{Funding:}

This study was funded by the China Medical Board (Grant Number: 14-194), Key Developing Discipline of the Shanghai Municipal Health Commission (Pediatrics) (Grant Number: 2016ZB0101), Shanghai municipal medical and health discipline construction projects (Pediatrics) (No. 2016ZB0104-01) and the Canadian Institutes of Health Research (Grant Number: CTP 87518).

\section{Availability of data and materials}

The data used and/or analyzed during the current study are available from the corresponding author on reasonable request.

Ethical approval: This study was approved by the Ethics Committee of the Children's Hospital of Fudan University.

Consent for publication: Not applicable.

Competing: No financial or nonfinancial benefits have been received or will be received from any party related directly or indirectly to the subject of this article.

Acknowledgements

The REIN-EPIQ Study Group: Yun Cao, Siyuan Jiang, Children's Hospital of Fudan Univeristy; Yong Ji, Children's Hospital of ShanXi / Women's Health Center of Shanxi, Shanxi, China; Shuping Han, Women's Hospital of Nanjing Medical University, Jiangsu, China; Sannan Wang, Suzhou Municipal Hospital, Jiangsu, China; Zhankui Li, Northwest Women and Children's Hospital, Shaanxi, China; Shiwen Xia, Women and Children's Hospital of Hubei Province, Hubei, China; Changyi Yang, Fujian Provincial 
Maternity and Children's Hospital/Affiliated Hospital of Fujian Medical University, Fujian, China; Chuanzhong Yang, The Affiliated Shenzhen Maternity and Child Healthcare Hospital of Southern Medical University, Guangdong, China; Ling Chen, Tongji Hospital, Tongji Medical College, Huazhong University of Science and Technology, Hubei, China; Ruobing Shan, Qingdao Women and Children's Hospital, Shandong, China; Ling Liu, Guiyang Maternal and Child Health Care Hospital, Guizhou, China; Bin Yi, Gansu Provincial Maternity and Child-care Hospital, Gansu, China; Zhenlang Lin, The 2nd Affiliated Hospital and Yuying Children's Hospital of Wenzhou Medical University, Zhejiang, China;Yang Wang, The First Affiliated Hospital of Anhui Medical University, Anhui, China; Jiangqin Liu, Shanghai First Maternity and Infant Hospital, Tongji University School of Medicine, Shanghai, China; Ling He, Jiangxi Provincial Children's Hospital, Jiangxi, China; Mingxia Li, MD, First Affiliated Hospital of Xinjiang Medical University, Xinjiang, China; Xinnian Pan, The Maternal and Child Health Hospital of Guangxi Zhuang Autonomous Region, Guangxi, China; Yan Guo, Children's Hospital of Nanjing Medical University, Jiangsu, China; Cuiqing Liu, Children's Hospital of Hebei Province, Hebei, China; Qin Zhou, The Affiliated Wuxi Maternity and Child Health Hospital of Nanjing Medical University, Jiangsu, China; Xiaoying Li, Qilu Children's Hospital of Shandong University, Shandong, China; Hong Xiong, Children's Hospital Affiliated to Zhengzhou University, Henan, China; Yujie Qi, Beijing Children's Hospital of Capital Medical University, Beijing, China; Mingyan Hei, The Third Xiangya Hospital of Central South University, Hunan, China

\section{References}

1. Chen C, Zhang JW, Xia HW, et al. Preterm Birth in China Between 2015 and 2016. Am J Public Health. 2019;109(11):1597-1604. doi:10.2105/AJPH.2019.305287.

2. Sun $L$, Yue H, Sun B, et al. Estimation of birth population-based perinatal-neonatal mortality and preterm rate in China from a regional survey in 2010. J Matern Fetal Neonatal Med. 2013;26(16):1641-1648. doi:10.3109/14767058.2013.794208

3. Asztalos E, Willan A, Murphy K, et al. Association between gestational age at birth, antenatal corticosteroids, and outcomes at 5 years: multiple courses of antenatal corticosteroids for preterm birth study at 5 years of age (MACS-5). BMC Pregnancy Childbirth. 2014;14:272. doi:10.1186/14712393-14-272

4. Roberts D, Brown J, Medley N, Dalziel SR. Antenatal corticosteroids for accelerating fetal lung maturation for women at risk of preterm birth. Cochrane Database Syst Rev. 2017;3(3): CD004454. doi:10.1002/14651858.CD004454.pub3

5. Briceño-Pérez C, Reyna-Villasmil E, Vigil-De-Gracia P. Antenatal corticosteroid therapy: Historical and scientific basis to improve preterm birth management. Eur J Obstet Gynecol Reprod Biol. 2019;234:32-37. doi:10.1016/j.ejogrb.2018.12.025

6. Skoll A, Boutin A, Bujold E, et al. No. 364-Antenatal Corticosteroid Therapy for Improving Neonatal Outcomes. J Obstet Gynaecol Can. 2018;40(9):1219-1239. doi:10.1016/j.jogc.2018.04.018

7. Committee on Obstetric Practice. Committee Opinion No. 713: Antenatal Corticosteroid Therapy for Fetal Maturation. Obstet Gynecol. 2017;130(2):e102-e109. doi:10.1097/AOG.0000000000002237 
8. Roberts D, Brown J, Medley N, Dalziel SR. Antenatal corticosteroids for accelerating fetal lung maturation for women at risk of preterm birth. Cochrane Database Syst Rev. 2017;3(3):CD004454. doi:10.1002/14651858.CD004454.pub3

9. Melamed N, Shah J, Soraisham A, et al. Association between antenatal corticosteroid administrationto-birth interval and outcomes of preterm neonates. Obstet Gynecol. 2015;125(6):1377-1384. doi:10.1097/AOG.0000000000000840

10. Travers $\mathrm{CP}$, Clark RH, Spitzer AR, et al. Exposure to any antenatal corticosteroids and outcomes in preterm infants by gestational age: prospective cohort study. BMJ. 2017;356: j1039. doi:10.1136/bmj.j1039

11. Fu H, Liu L, Zhang J, Wang Q. Clinical features, prevention and treatment of respiratory distress syndrome in neonates of different gestational ages in tertiary hospitals in Northwest China. Chinese Journal of Contemporary Pediatrics. 2015; (10):1039-1044. doi:10.7499/j.issn.10088830.2015.10.004

12. Collaboratives for epidemiological investigation of respiratory distress syndrome in preterm infants. Effect of antenatal corticosteroids therapy on the mortality and morbidity of small for gestational age infants born at 24-34 completed weeks: a retrospective multicenter study. Chinese Journal of Pediatrics. 2017;55(8):613-618. doi:10.3760/cma.j.issn.0578-1310.2017.08.013

13. Jiang S, Yang Z, Shan R, et al. Neonatal Outcomes Following Culture-negative Late-onset Sepsis Among Preterm Infants. Pediatr Infect Dis J. 2020;39(3):232-238.

doi:10.1097/INF.0000000000002558

14. John K G, Dimitrios D, Kalpesh P. Assessing Outcome in Interhospital Infant Transport: The Transport Risk Index of Physiologic Stability Score at Admission. 2012;29(7):509-514. doi:10.1055/s-00321310521

15. Shennan AT, Dunn MS, Ohlsson A, et al. Abnormal pulmonary outcomes in premature infants: prediction from oxygen requirement in the neonatal period. Pediatrics. 1988;82: 527-532. doi: http://dx.doi.org/

16. Bell MJ, Ternberg JL, Feigin RD, et al. Neonatal necrotizing enterocolitis. Therapeutic decisions based upon clinical staging. Ann Surg. 1978;187:1-7. doi: 10.1097/00000658-197801000-00001

17. Papile LA, Burstein J, Burstein R, et al. Incidence and evolution of subependymal and intraventricular hemorrhage: a study of infants with birth weights less than 1,500gm. J Pediatr. 1978;92:529-534. doi: 10.1016/S0022-3476(78)80282-0

18. Jiang S, Hong L, Gai J, et al. Early-onset Sepsis Among Preterm Neonates in China, 2015 to 2018. Pediatr Infect Dis J. 2019;38(12):1236-1241. doi:10.1097/INF.0000000000002492

19. The Committee for the Classification of Retinopathy of Prematurity. An international classification of retinopathy of prematurity. Arch Ophthalmol. 1984;102:1130-1134

20. Fanaroff AA, Stoll BJ, Wright LL, et al. Trends in neonatal morbidity and mortality for very low birthweight infants. Am J Obstet Gynecol. 2007;196:147 e1-8.17. doi:10.1016/j.ajog.2006.09.014 
21. Ancel PY, Goffinet F, Group E-W, et al. Survival and morbidity of preterm children born at 22 through 34 weeks' gestation in France in 2011: results of the EPIPAGE-2 cohort study. JAMA Pediatr. 2015; 169: 230-8. doi:10.1001/jamapediatrics.2014.3351.

22. Brownfoot FC, Gagliardi DI, Bain E, et al. Different corticosteroids and regimens for accelerating fetal lung maturation for women at risk of preterm birth. Cochrane Database Syst Rev. 2013;(8): CD006764. doi:10.1002/14651858.CD006764.pub3

23. Crowther CA, Ashwood P, Andersen CC, et al. Maternal intramuscular dexamethasone versus betamethasone before preterm birth (ASTEROID): a multicentre, double-blind, randomised controlled trial. Lancet Child Adolesc Health. 2019;3(11):769-780. doi:10.1016/S2352-4642(19)30292-5

24. Melamed N, Asztalos E, Murphy K, et al. Neurodevelopmental disorders among term infants exposed to antenatal corticosteroids during pregnancy: a population-based study. BMJ Open. 2019;9(9):e031197. doi:10.1136/bmjopen-2019-031197

25. Finken MJ, Keijzer-Veen MG, Dekker FW, et al. Antenatal glucocorticoid treatment is not associated with long-term metabolic risks in individuals born before 32 weeks of gestation. Arch Dis Child Fetal Neonatal Ed. 2008;93(6):F442-F447. doi:10.1136/adc.2007.128470

26. Crowther CA, Anderson PJ, McKinlay CJ, et al. Mid-Childhood Outcomes of Repeat Antenatal Corticosteroids: A Randomized Controlled Trial. Pediatrics. 2016;138(4):e20160947. doi:10.1542/peds.2016-0947

27. Wapner RJ, Sorokin Y, Mele L, et al. Long-term outcomes after repeat doses of antenatal corticosteroids. N Engl J Med. 2007;357(12):1190-1198. doi:10.1056/NEJMoa071453

28. Levin HI, Ananth CV, Benjamin-Boamah C, et al. Clinical indication and timing of antenatal corticosteroid administration at a single center. BJOG. 2016;123(3):409-414. doi:10.1111/14710528.13730

29. Chandiramani M, Di Renzo GC, Gottschalk E, et al. Fetal fibronectin as a predictor of spontaneous preterm birth: a European perspective. J Matern Fetal Neonatal Med. 2011;24(2):330-336. doi:10.3109/14767058.2010.496879

30. Swales DA, Grande LA, Wing DA, et al. Can Placental Corticotropin-Releasing Hormone Inform Timing of Antenatal Corticosteroid Administration? J Clin Endocrinol Metab. 2019;104(2):443-450. doi:10.1210/jc.2018-00956

31. Shigemi D, Yasunaga H. Antenatal corticosteroid administration in women undergoing tocolytic treatment who delivered before 34 weeks of gestation: a retrospective cohort study using a national inpatient database. BMC Pregnancy Childbirth. 2019;19(1):17. Published 2019 Jan 9. doi:10.1186/s12884-019-2174-1

32. Profit J, Goldstein BA, Tamaresis J, et al. Regional variation in antenatal corticosteroid use: a network-level quality improvement study. Pediatrics. 2015;135(2): e397. doi:10.1542/peds.20142177

33. Lee SK, McMillan DD, Ohlsson A, et al. Variations in practice and outcomes in the Canadian NICU network: 1996-1997. Pediatrics. 2000;106(5):1070-1079. doi:10.1542/peds.106.5.1070 
34. Haslam MD, Lisonkova S, Creighton D, et al. Severe Neurodevelopmental Impairment in Neonates Born Preterm: Impact of Varying Definitions in a Canadian Cohort. J Pediatr. 2018;197:75-81.e4. doi:10.1016/j.jpeds.2017.12.020

35. Katarzyna K, Iwona S, Paulina U, et al. Relation between time interval from antenatal corticosteroids administration to delivery and neonatal outcome in twins. J Obstet Gynaecol Res. 2016;42(6):625631. doi:10.1111/jog.12966

36. Kaplan HC, Sherman SN, Cleveland C, et al. Reliable implementation of evidence: a qualitative study of antenatal corticosteroid administration in Ohio hospitals. BMJ Qual Saf. 2016;25:173-81. doi:10.1136/bmjqs-2015-003984

37. Profit J, Goldstein BA, Tamaresis J, et al. Regional variation in antenatal corticosteroid use: a network-level quality improvement study. Pediatrics. 2015;135(2):e397-e404. doi:10.1542/peds.2014-2177

38. Howson C, Kinney M, Lawn J, editor. March of Dimes, PMNCH, Save the Children, WHO(2012) Born Too Soon: the global action report on preterm birth. Geneva: World Health Organization; 2012.

39. Liu G, Segrè J, Gülmezoglu A, et al. Antenatal corticosteroids for management of preterm birth: a multi-country analysis of health system bottlenecks and potential solutions. BMC Pregnancy Childbirth. 2015;15(2):S3. doi:10.1186/1471-2393-15-S2-S3

\section{Figures}




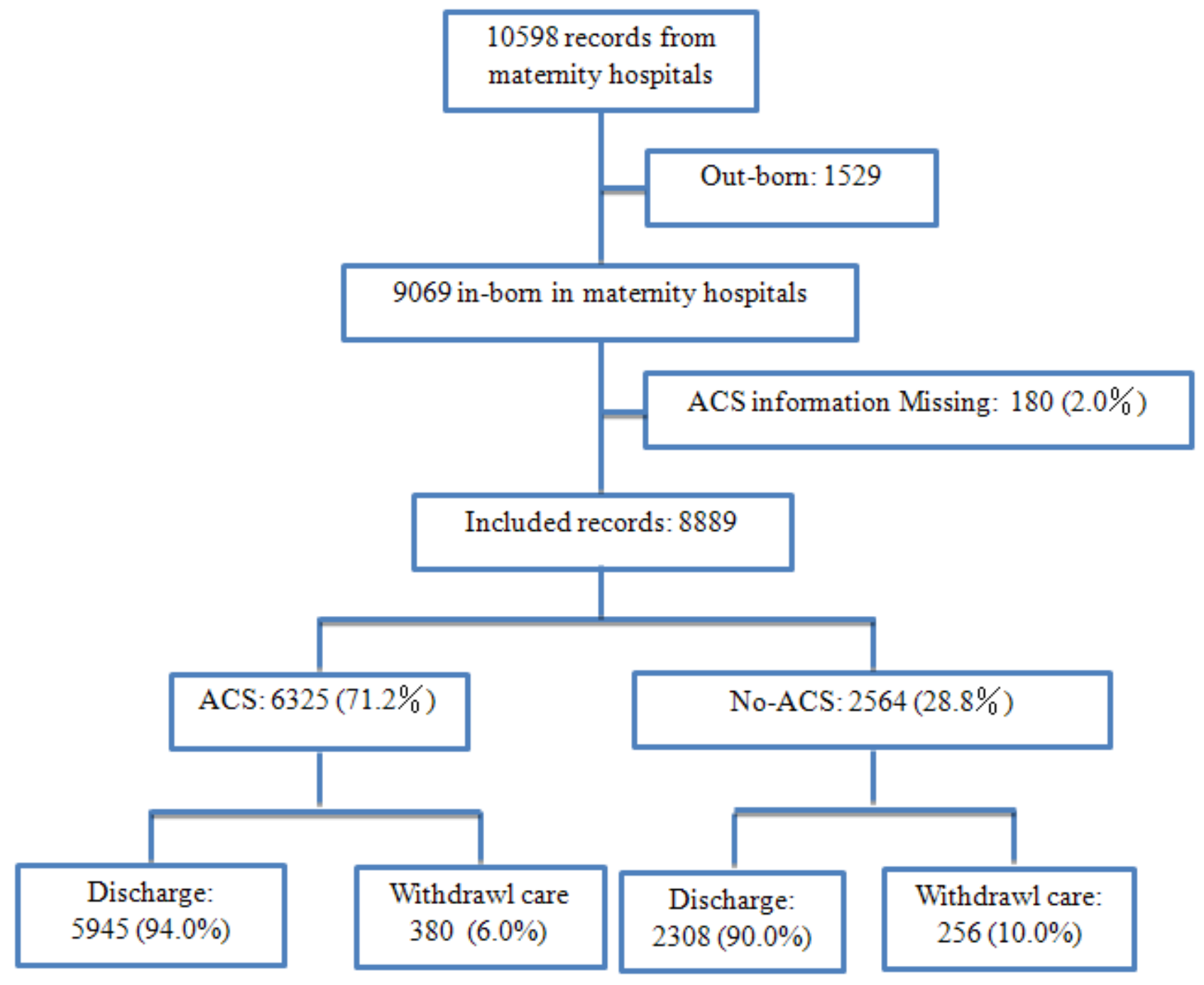

Figure 1

Flow diagram of research cohort. ACS: antenatal corticosteroids. 


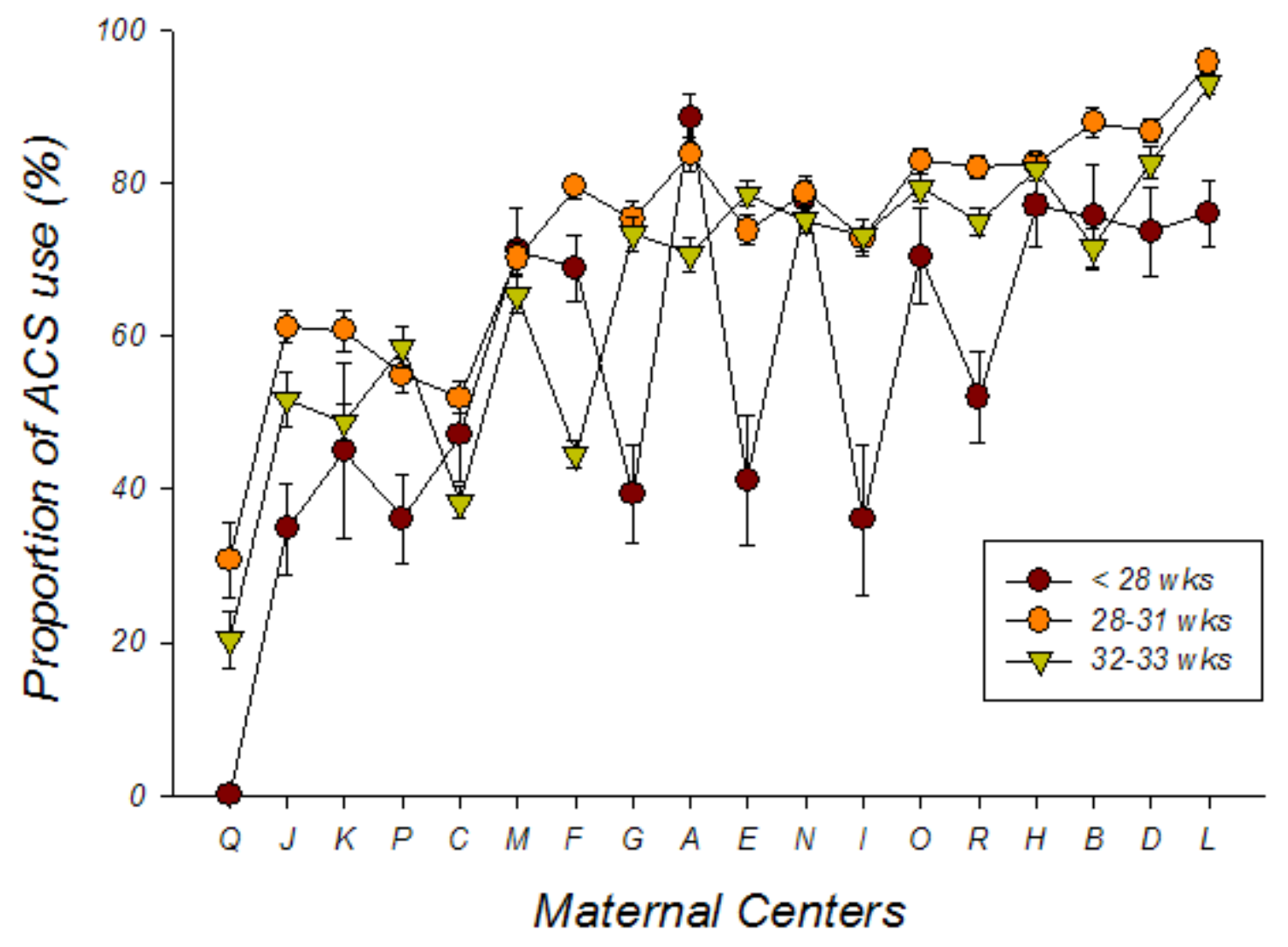

Figure 2

Proportion of antenatal corticosteroids (ACS, y axis) in different maternal centers (x axis) on preterm infants of less than 28 weeks (dark red), 28-31 weeks (orange) and 32-33 weeks of gestational age (green). 